\title{
Orbital Approximation for the Reduced Bloch Equations: Fermi-Dirac Distribution for Interacting Fermions and Hartree-Fock Equation at Finite Temperature
}

\author{
Liqiang Wei \\ Institute for Theoretical Atomic, Molecular and Optical Physics \\ Harvard University, Cambridge, MA 02318 \\ Chiachung Sun \\ Institute of Theoretical Chemistry, Jilin University \\ Changchun, Jilin 130023 P. R. China
}

November 2, 2018

\begin{abstract}
In this paper, we solve a set of hierarchy equations for the reduced statistical density operator in a grand canonical ensemble for an identical many-body fermion system without or with two-body interaction. We take the single-particle approximation, and obtain an eigen-equation for the single-particle states. For the case of no interaction, it is an eigen-equation for the free particles, and solutions are therefore the plane waves. For the case with two-body interaction, however, it is an equation which is the extension of usual Hartree-Fock equation at zero temperature to the case of any finite temperature. The average occupation number for the single-particle
\end{abstract}


states with mean field interaction is also obtained, which has the same Fermi-Dirac distribution form as that for the free fermion gas. The derivation demonstrates that even for an interacting fermion system, only the lowest $N$ orbitals, where $N$ is the number of particles, are occupied at zero temperature. In addition, their practical applications in such fields as studying the temperature effects on the average structure and electronic spectra for macromolecules are discussed.

$\underline{P A C S}$ 05.30.-d; 31.70.-f; 71.10.-w; 72.80.Le

\section{Introduction}

Quantum statistical mechanics provides a most general theoretical framework for studying the structure and dynamics of an interacting many-body system. Combined with mathematical laws of statistical distribution, it investigates the microscopic quantum states of a system with many degrees of freedom, its corresponding macroscopic thermodynamic behaviors, and their

interplay [1-4]. It covers the usual quantum mechanics as a special case at zero temperature [5].

Experimental investigation of temperature or pressure effects on the electronic structure and related spectra for molecules or solids has been for some time [6-8]. However, a complete or systematic theoretical work has not been developed and seen in the literature. The quantum chemistry at finite temperature or pressure is not a well-defined or well-established field [9]. Indeed, the effect of temperature or pressure on microscopic structure is a complicated phenomenon. There exists different functioning mechanisms. One consideration is that the variation of the temperature, according to 
the Fermi-Dirac statistics, will change the thermal probability distribution of single-particle states for a free electron gas. Similar situation should be expected to occur for an interacting electron system, and therefore its microscopic structure will be correspondingly altered. Another consideration is that, for molecules or solids, the thermal excitation will cause the change of the time scales for the molecular motions. This will most likely bring about the transitions of electronic states, and therefore lead to the breakdown of the Born-Oppenheimer approximation. Electron-phonon interaction is a fundamental topic in solid state physics and its temperature dependence is well-known. In this article, we tackle the issues in a simpler way. We treat only an interacting identical fermion system, or neglect the coupling of the electronic motion with those of the nucleus in molecules or solids. We expect that some sort of the general conclusions will come out from this study. As a matter of fact, this is also the approach usually adopted in non-adiabatic molecular dynamics, in which purely solving the eigenequation for the electrons will provide the reference states for investigating the coupling motions between the electrons and the nucleus of the molecules.

In a recent paper [10], we have deduced a set of hierarchy equations for the reduced statistical density operators in both canonical and grand canonical ensembles. They provide a law according to which the reduced density matrix varies in temperature. They also offer a route for a direct determination of the reduced density operator for the statistical ensembles.

This paper is an initial endeavor, on the basis of the equations we derived in paper [10], to investigate the issues related to the interplay between the microscopic structure and macroscopic observables, and specifically, the tem- 
perature effects on the electronic structure of molecules or solids. We take the orbital model and solve the equations for the grand canonical ensemble in an approximate way. In the next Section, we first consider the case of a free electron gas, and evaluate the terms related to the single-particle operator of the reduced Bloch equations, we get the solutions of a plane wave as well as the usual Fermi-Dirac statistics of a free electron gas. In Section 3, we solve the equations for the identical fermion system with two-body interaction. We obtain an energy equation for the single-particle states which is the extension of usual Hartree-Fock equation at zero temperature to the case of any finite temperature. The average occupation number is also obtained which has the same form as that for a free fermion gas, which is the Fermi-Dirac distribution. The Section 4 is a summary and conclusion. The implications and possible applications are discussed.

\section{Free Fermion Gas}

In this and next sections, we consider the solution of reduced Bloch equations for the grand canonical ensemble we derived in paper [10] as shown below,

$$
\begin{aligned}
-\frac{\partial}{\partial \beta} D^{p}= & \bar{H}_{1}^{p} D^{p}+(p+1) L_{p+1}^{p}\left[\bar{h}(p+1) D^{p+1}\right]+(p+1) L_{p+1}^{p}\left[\sum_{i=1}^{p} g(i, p+1) D^{p+1}\right]+ \\
& +\left(\begin{array}{c}
p+2 \\
2
\end{array}\right) L_{p+2}^{p}\left[g(p+1, p+2) D^{p+2}\right]
\end{aligned}
$$

where

$$
\bar{H}_{1}^{p}=\sum_{i=1}^{p} \bar{h}(i)+\sum_{i<j}^{p} g(i, j),
$$


and

$$
\bar{h}(i)=h(i)-\mu \text {. }
$$

The $h(i)$ and $g(i, j)$ are one- and two-body operators, and the rest of symbols are defined in [10]. We choose a grand canonical ensemble for our study because we want to compare our results with those already known for a free fermion gas. Just like solving any other equations of motion, the exact solutions can be obtained only for very few cases. Some sort of approximations have to be made. Nevertheless, more universal conclusions might be possibly obtained. A common approximation scheme is to truncate the hierarchy. Depending on the specific physical situation, this is accomplished by approximating the higher-order density matrix as a functional of the lower-order ones. In this paper, the system considered is $N$-fermions and the decoupling scheme we assume is to express $D^{p+1}$ by $D^{p}$ in the following way [11-15],

$$
\begin{aligned}
D^{p+1} & =D^{p} \wedge D^{1} / D^{0} \\
& =\underbrace{D^{1} \wedge D^{1} \wedge \ldots \wedge D^{1}}_{p+1} /\left(D^{0}\right)^{p},
\end{aligned}
$$

and

$$
\begin{aligned}
D^{p+2} & =D^{p+1} \wedge D^{1} / D^{0} \\
& =\underbrace{D^{1} \wedge D^{1} \wedge \ldots \wedge D^{1}}_{p+2} /\left(D^{0}\right)^{p+1} .
\end{aligned}
$$

That is, the $p$ th-order reduced density matrix can be expressed as a $p$-fold Grassmann product of its first-order reduced density matrices. This implies that we have taken an independent-particle approximation. To proceed further, we need to evaluate various terms of right hand side of Eq. (1). 
We first study the case without any interaction, where we only need to calculate the first and second terms of Eq. (1). For the first term,

$$
\begin{aligned}
\bar{H}_{1}^{p} \cdot D^{p} & =\bar{H}_{1}^{p} \cdot \underbrace{D^{1} \wedge D^{1} \wedge \ldots \wedge D^{1}}_{p} /\left(D^{0}\right)^{p-1} \\
& =\sum_{i=1}^{p} \underbrace{D^{1} \wedge D^{1} \wedge \ldots \wedge \bar{h}_{(i)} \cdot D^{1} \wedge \ldots \wedge D^{1}}_{p} /\left(D^{0}\right)^{p-1} .
\end{aligned}
$$

For the second term, the calculation is more complex, and the following formulae related to the decomposition of a permutation group in terms of its subgroups are needed [16],

$$
\begin{aligned}
A_{p+1} & =\frac{1}{p+1}\left[A_{p}-\sum_{i=1}^{p} A_{p} \cdot(p+1, i)\right] \\
& =\frac{1}{p+1}\left[A_{p}-\sum_{i=1}^{p}(p+1, i) \cdot A_{p}\right]
\end{aligned}
$$

and

$$
\left.\bar{h}(p+1) \cdot A_{p} \cdot(p+1, i)=A_{p} \cdot(p+1, i) \cdot\right] \bar{h}(i),
$$

where $A_{p}$ is a $p$ th-order antisymmetric operator, and $(p+1, i)$ is an exchange between $p+1$ and $i$. Therefore,

$$
\begin{aligned}
& (p+1) L_{p+1}^{p}\left[\bar{h}(p+1) D^{p+1}\right] \\
= & (p+1) L_{p+1}^{p}\left[\bar{h}(p+1) A_{p+1}(\underbrace{D^{1} \otimes D^{1} \otimes \ldots \otimes D^{1}}_{p+1}) A_{p+1}\right] /\left(D^{0}\right)^{p} \\
= & \left(\operatorname{Tr} \bar{h} D^{1}\right) \underbrace{D^{1} \wedge D^{1} \wedge \ldots \wedge D^{1}}_{p} /\left(D^{0}\right)^{p}- \\
& -\sum_{i=1}^{p} \underbrace{D^{1} \wedge \ldots \wedge D^{1} \bar{h}_{(i)} D^{1} \wedge \ldots \wedge D^{1} /\left(D^{0}\right)^{p} .}_{p}
\end{aligned}
$$

For the special case of $p=1$, inserting Eqs. (5) and (8) into Eq. (1) yields

$$
-\frac{\partial}{\partial \beta} D^{1}=\bar{h} D^{1}+\frac{\operatorname{Tr}\left(\bar{h} D^{1}\right)}{D^{0}} D^{1}-\frac{1}{D^{0}} D^{1} \bar{h} D^{1} .
$$


This is the Bloch equation for the first-order reduced density matrix of an identical particle system with no interaction under single-particle approximation.

Define

$$
\rho^{1}=D^{1} / D^{0},
$$

and then Eq. (9) can be simplified as

$$
-\frac{\partial}{\partial \beta} \rho^{1}=\bar{h} \rho^{1}-\rho^{1} \bar{h} \rho^{1} .
$$

From above equation and its conjugate, we get

$$
h \rho^{1}-\rho^{1} h=0 .
$$

This means that $h$ and $\rho^{1}$ commute. Since they are also Hermitian, they have common eigenfunctions $\left\{\left|\phi_{i}\right\rangle\right\}$, which are plane waves,

$$
h\left|\phi_{i}>=\epsilon_{i}\right| \phi_{i}>,
$$

and

$$
\rho^{1}\left|\phi_{i}>=\omega\left(\beta, \mu, \epsilon_{i}\right)\right| \phi_{i}>
$$

with

$$
\rho^{1}=\sum_{i} \omega\left(\beta, \mu, \epsilon_{i}\right)\left|\phi_{i}><\phi_{i}\right| .
$$

Substituting Eq. (15) into Eq. (11), we can obtain the equation the thermal probability $\omega\left(\beta, \mu, \epsilon_{i}\right)$ satisfies,

$$
-\frac{\partial}{\partial \beta} \omega\left(\beta, \mu, \epsilon_{i}\right)=\left(\epsilon_{i}-\mu\right) \omega\left(\beta, \mu, \epsilon_{i}\right)-\left(\epsilon_{i}-\mu\right) \omega^{2}\left(\beta, \mu, \epsilon_{i}\right) .
$$

Its solution takes the form,

$$
\omega\left(\beta, \mu, \epsilon_{i}\right)=\frac{1}{1+e^{\beta\left(\epsilon_{i}-\mu\right)}},
$$


which is just the usual Fermi-Dirac distribution. Therefore, by directly solving the reduced Bloch equations for the reduced density matrix in a grand canonical ensemble under orbital approximation, we not only have obtained the eigen solutions of a plane wave but also have recovered the usual FermiDirac distribution for the free electron gas.

\section{Hartree-Fock Equation at Finite Tempera- ture}

In this section, we solve the reduced Bloch equation (1) for the case of an identical fermion system with two-body interaction under the orbital approximation (4).The first term and the second term remain the same as those for the free fermion case. We need to evaluate the remaining two terms. In particular, for $p=1$, the Eq. (1) reads

$-\frac{\partial}{\partial \beta} D^{1}=\bar{h} D^{1}+\frac{\operatorname{Tr}\left(\bar{h} D^{1}\right)}{D^{0}} D^{1}-\frac{1}{D^{0}} D^{1} \bar{h} D^{1}+2 L_{2}^{1}\left[g(1,2) D^{2}\right]+3 L_{3}^{1}\left[g(2,3) D^{3}\right]$.

The last two terms can be evaluated in a straightforward way, which yields

$$
2 L_{2}^{1}\left[g(1,2) D^{2}\right]=(J-K) D^{1},
$$

and

$$
3 L_{3}^{1}\left[g(2,3) D^{3}\right]=\frac{\operatorname{Tr}\left(g D^{2}\right)}{D^{0}}-\frac{1}{D^{0}} D^{1}(J-K) D^{1}
$$

where

$$
J=\operatorname{Tr}_{2}\left[g \cdot D^{1}(2 ; 2)\right] / D^{0}
$$

and

$$
K=\operatorname{Tr}_{2}\left[g \cdot(2,3) \cdot D^{1}(2 ; 2)\right] / D^{0}
$$


are called the Coulomb and exchange operators, respectively. The action of $K$ is

$$
\begin{aligned}
K \cdot D^{1}(3 ; 3) & =\operatorname{Tr}_{2}\left[g \cdot(2,3) \cdot D^{1}(2 ; 2)\right] / D^{0} \cdot D^{1}(3 ; 3) \\
& =\operatorname{Tr}_{2}\left[g \cdot D^{1}(3 ; 2) \cdot D^{1}(2 ; 3)\right] / D^{0} .
\end{aligned}
$$

Substitution of Eqs. (19) and (20) into Eq. (18) yields the Bloch equation for the first-order reduced density matrix of $N$ interacting fermions under orbital approximation,

$$
-\frac{\partial}{\partial \beta} D^{1}=(F-\mu) D^{1}+\left(\frac{\operatorname{Tr} \bar{h} D^{1}}{D^{0}}+\frac{\operatorname{Tr} D^{2}}{D^{0}}\right) D^{1}-\frac{1}{D^{0}} D^{1}(F-\mu) D^{1},
$$

where

$$
F=h+J-K,
$$

is called the Fock operator at finite temperature. Redefine the normalized first-order reduced density operator

$$
\rho^{1}=D^{1} / D^{0}
$$

we can simply above equation into

$$
-\frac{\partial}{\partial \beta} \rho^{1}=(F-\mu) \rho^{1}-\rho^{1}(F-\mu) \rho^{1} .
$$

From Eq. (27) and its conjugate, we get

$$
F \rho^{1}-\rho^{1} F=0,
$$

which means that the Fock operator $F$ and $\rho^{1}$ commute. They are also Hermitian, and therefore they have common eigenvectors $\left\{\mid \phi_{i}>\right\}$. These vectors are determined by the following eigen equation for the Fock operator,

$$
F\left|\phi_{i}>=\epsilon_{i}\right| \phi_{i}>\text {. }
$$


The first-order reduced density operator is correspondingly expressed as

$$
\rho^{1}=\sum_{i} \omega\left(\beta, \mu, \epsilon_{i}\right)\left|\phi_{i}><\phi_{i}\right|
$$

where $\omega\left(\beta, \mu, \epsilon_{i}\right)$ is the thermal probability that the orbital is found to be in

the state $\left\{\left|\phi_{i}\right\rangle\right\}$ at finite temperature T. Substituting Eq. (30) into Eq. (27), we can obtain the equation this thermal probability $\omega\left(\beta, \mu, \epsilon_{i}\right)$ satisfies,

$$
-\frac{\partial}{\partial \beta} \omega\left(\beta, \mu, \epsilon_{i}\right)=\left(\epsilon_{i}-\mu\right) \omega\left(\beta, \mu, \epsilon_{i}\right)-\left(\epsilon_{i}-\mu\right) \omega^{2}\left(\beta, \mu, \epsilon_{i}\right) .
$$

Its solution has the same usual form of Fermi-Dirac statistics for the free electron gas as follows,

$$
\omega\left(\beta, \mu, \epsilon_{i}\right)=\frac{1}{1+e^{\beta\left(\epsilon_{i}-\mu\right)}},
$$

with energy levels $\left\{\epsilon_{i}\right\}$ determined by Eq. (29).

\section{Discussions and Conclusions}

In this paper, we have solved the set of hierarchy Bloch equations for the reduced statistical density operator in a grand canonical ensemble under single-orbital approximation for the identical fermion system without or with two-body interaction. For the case without any interaction, we not only get a plane wave solution for the eigenstates and eigenvalues, but also recover the usual Fermi-Dirac distribution of a free electron gas. For the situation with two-body interaction, we obtain an eigen-equation for the single-particle states. It is the extension of usual commonly used Hartree-Fock equation at the absolute zero temperature to the case of any finite temperature. The 
average occupation number formula for each single-particle state is also obtained, which has the same analytical form as that for the free electron gas with the single-particle state energy determined by the Hartree-Fock equation at finite temperature (29).

From Eqs. (21), (22) and (30), we see that the Coulomb operator $J$, the exchange operator $K$, and therefore the Fock operator $F$ are both the coherent and the incoherent superpositions of single-particle states. They are all temperature-dependent through an incoherent superposition factor, the Fermi-Dirac distribution, $\omega\left(\beta, \mu, \epsilon_{i}\right)$. Therefore, the mean force and corresponding microscopic structure are temperature-dependent.

It is easy to see that, when temperature is zero, only $N$ single-particle states with energy levels small than the chemical potential $\mu$ are occupied as for the free electron gas. That is, the fact that only lowest $N$ orbitals (or holes) are occupied holds also for the interacting case. Therefore, only a single determinant wavefunction is enough for a description of interacting $N$-particle wavefunctions. Say in another way, there exists a correspondingly effective and strict one-particle state description of an interacting $N$-particle system. This is also the spirit of current density functional theory [17], and is consistent with the third law of thermodynamics.

Even though we have simplified the structure issue for a molecule or a solid at finite temperature, the equations we obtained in this paper will find wide applications. The examples include the investigation of temperature effects on the average structure and the electronic spectra of macromolecules and the study of conduction electrons in metals and so forth [18-21]. 


\section{References}

[1] R. C. Tolman, The Principles of Statistical Mechanics (Oxford University Press, 1938).

[2] R. P. Feynman, Statistical Mechanics (W. A. Benjamine, Reading, Mass., 1972).

[3] D. Chandler, Introduction to Modern Statistical Mechanics (Oxford University, 1987).

[4] K. Huang, Statistical Mechanics (John Wiley \& Sons, 2nd Edition, 1987).

[5] P. O. Löwdin, Intern. J. Quantum Chem. Vol xxix, 1651 (1986).

[6] K. Pichler, D. A. Halliday, D. D. C. Bradley, P. L. Burn, R. H. Friend, and A. B. Holmes, J. Phys.: Condens. Matter 5, 7155 (1993).

[7] W. Klemperer, Ann. Rev. Phys. Chem. 46, 1 (1995).

[8] H. G. Drickamer and C. W. Franck, Electronic Transitions and the High Pressure Chemistry and Physics of Solids (London, Chapman and Hall, New York, 1973).

[9] S. P. Kwasniewski, J. P. Francois, and M. S. Deleuze, J. Phys. Chem A 107, 5168 (2003).

[10] L. Wei and C. C. Sun, submitted (2003); e-print, cond-mat/0306306.

[11] P. O. Löwdin, Phys. Rev. 97, 1474 (1955). 
[12] L. Cohen and C. Frishberg, Phys. Rev. A 13, 927 (1976).

[13] H. Nakatsuji, Phys. Rev. A 14, 41 (1976).

[14] H. Schlosser, Phys. Rev. A 15, 1349 (1977).

[15] J. E. Harriman, Phys. Rev. A 19, 1893 (1979).

[16] I. G. Kaplan, Symmetry of Many Electron Systems (Academic Press, New York, 1975).

[17] W. Kohn and L. J. Sham, Phys. Rev. A 140, 1133 (1965).

[18] J. Yu, M. Hayashi, S. H. Lin, K.-K. Liang, J. H. Hsu, W. S. Fann, C.-I. Chao, K.-R. Chuang, S.-A. Chen, Synth. Met. 82, 159 (1996).

[19] E. Peeters, A. M. Ramos, S. C. J. Meskers, and R. A. J. Janssen, J. Chem. Phys. 112, 9445 (2000).

[20] S. P. Kwasniewski, J. P. Francois, and M. S. Deleuze, Int. J. Quantum Chem. 85, 557 (2001).

[21] J. Gierschner, Hans-Georg Mack, L. Lüer, and D. Oelkrug, J. Chem. Phys. 116, 8596 (2002). 